\title{
EL PENSAMIENTO CRÍTICO EN LA EDUCACIÓN UNIVERSITARIA
}

\author{
MARCELO VARELA \\ Universidad Central del Ecuador
}

Recepción manuscrito: 1 de julio de 2014

Aceptación versión final: 30 de octubre de 2014

\begin{abstract}
RESUMEN El pensamiento crítico es una herramienta ausente en la educación superior en donde docentes y estudiantes están lejos de utilizar esta herramienta cognitiva. Las formas de fomentar el pensamiento crítico proliferan a través de conferencias e iniciativas educativas; sin embargo, los esfuerzos pedagógicos en esta área están basados en concepciones erróneas. Aquí se analiza la necesidad de una concepción defendible del pensamiento crítico y una cuenta perspicaz de las características o cualidades necesarias para ser un pensador crítico.

palabras ClaVe Pensamiento crítico, pedagogía, enseñanza, docentes, estudiantes.
\end{abstract}

ABSTRACT Critical thinking is an almost absent tool within higher education, where professors and students are far from using it as a cognitive tool. The ways to encourage critical thinking spread through lectures and academic initiatives. However, the pedagogical efforts in this area are based on erroneous conceptions. This work analyzes the need of a critical thinking defendable conception and a sharp list of the necessary features or qualities required to be a critical thinker.

KEY WORDS Critical thinking, education, teaching, teachers, students.

JEL CODE A2O, A22, I21

\section{CONCEPCIÓN DEL PENSAMIENTO CRÍTICO}

Mientras menos se entiende un sistema, la gente tiende a usar una mayor cantidad de datos para describirlo.

R. Ackoff

El pensamiento crítico es conceptualizado frecuentemente en términos de habilidades, procesos, procedimientos y práctica. Gran parte de la literatura educativa se refiere a las habilidades cognitivas o de pensamiento, y en otros casos iguala el pensamiento crítico con determinados procesos mentales o movimientos procesales que se pueden mejorar con la práctica (Bailin et al., 1999). 
A pesar de lo dicho anteriormente, ninguna concepción del pensamiento crítico debe construirse para capturar la mayor parte de lo que la gente tiene en mente cuando afirman que el desarrollo del pensamiento crítico es un objetivo importante de la educación. Es decir, tiene que ser verdad en el significado central de concepto básico del educador. En este sentido, ¿cuál es, entonces, el pensamiento crítico que generalmente tienen en mente los educadores cuando hablan de este concepto? Tal vez la mejor manera de acercarse a esta pregunta es considerar qué tipo de pensamiento crítico tienen los educadores. Esto sugiere que el pensamiento considerado como el pensamiento crítico debe ser dirigido hacia un fin o propósito, como respuesta a una pregunta, que permita la resolución de un problema, la elaboración de un plan, o la realización de un proyecto.

De esta manera, el pensamiento como crítica no solo supone que puede llegar a ser una crítica per se, ya que el pensamiento ha sido descuidado, y por ende la mayoría de los defensores del pensamiento crítico no concuerdan que este sea el pensamiento crítico. Por un lado, esto sugiere que debe cumplir con las normas apropiadas de pensar, si es considerado pensamiento crítico. Por otra parte, estas normas no pueden ser satisfechas sólo por accidente o casualidad, sino que deben estar presentes en el diario vivir, y se lo puede obtener a través de la abstracción.

Así, para Bailin et al. (1999b), el pensamiento crítico normalmente es entendido por los educadores que tienen por lo menos estas tres características: 1) Se hace con el propósito de recuperar la mente sobre lo que debe creer o hacer, 2) La persona que se ocupa en el pensamiento está tratando de precisar los estándares de adecuación y precisión en el pensamiento, y 3) Pensar las normas pertinentes de algún nivel. Es decir, las normas pertinentes en el pensamiento son supuestas porciones de un todo o nada, por eso el pensamiento crítico habla de buenos y malos pensamientos para indicar el grado limitante de los signos característicos pertinentes del pensamiento de una persona. Por esta razón, el concepto de pensamiento básico critico de Bailin et al. (1999a) es esencialmente una noción normativa, es decir que el pensamiento crítico es una buena sensación, es la cualidad del pensamiento, no los procesos que distingue al pensamiento crítico. Además para decidir cómo se describe las actividades y normas del pensamiento crítico, se necesita describir a éste, es decir, qué tipo de tareas abarca el pensamiento crítico.

Siguiendo a Brookfield (1987), el pensamiento crítico comprende algunas características: 1) Es una actividad productiva y positiva, 2) Es un proceso, no un resultado, 3) Las manifestaciones pueden variar de acuerdo a los contextos en que se produce, 4) Es desencadenada por eventos y negativos positivos, y 5) Es emotivo y racional. Además, estas características debe incluir algunos componentes: 1) Identificar suposiciones difíciles, 2) Desafiar la importancia del contexto, 3) Los pensadores críticos tratan de imaginar y explorar alternativas, 4) Imaginar y explorar alternativas conduce al escepticismo reflexivo. Lo que nos permite indicar que el pensamiento crítico requiere a menudo imaginar posibles consecuencias, generando enfoques generales y la identificación de puntos de vista alternativos. Por lo tanto, la creatividad juega un importante papel en el pensamiento crítico.

Generalmente el pensamiento crítico se reduce a una evaluación de los productos intelectuales, incluyendo declaraciones, argumentos y razones como componentes importantes del pensamiento crítico, por tanto, el concebirlo como actividades excesivamente estrechas 
produce un error de gran magnitud, puesto que el pensamiento crítico debe ser más que una simple evaluación de productos intelectuales; ya que el pensamiento crítico tiene lugar en el contexto de la discusión y el diálogo. Además de la evaluación apropiada de los productos intelectuales, el pensamiento crítico responde de manera constructiva a las razones y a los argumentos dados por otros en el contexto de la discusión.

\section{PENSAMIENTO CRÍTICO COMO HABILIDAD}

Muchos educadores y teóricos parecen ver la tarea de enseñar sin el pensamiento crítico como una cuestión de desarrollar habilidades de pensamiento mediante la simple evaluación de productos intelectuales ${ }^{1}$. De hecho, el trabajo sobre el pensamiento está impregnado de habilidad del habla. Los principales teóricos en el área del pensamiento crítico conceptualizan el pensamiento crítico en gran medida en términos de habilidad. Es evidente que ser un pensador crítico implica, entre otras cosas, tener una cierta dosis de know-how. Comúnmente se piensa que estos pensadores son hábiles, por tanto, deben ser capaces de cumplir con las normas pertinentes del buen pensamiento. Sin embargo, hay una fuerte tendencia entre los educadores para dividir las metas u objetivos educativos en tres tipos distintos: a) conocimientos, b) habilidades y c) actitudes (es decir, valores), y asignar el pensamiento crítico a la categoría de habilidades. Quien es hábil para captar la materia, es inteligente y por ende tiene pensamiento crítico, pero esto es una falacia, ya que la comprensión del pensamiento crítico es diferente al solo hecho de evaluación.

Entonces, la concepción del pensamiento crítico como una habilidad en este sentido implica más que un individuo sea un pensador competente o perito. Se basa en una concepción de la habilidad como una operación de identificación que es genérica y discreta. El pensamiento crítico implica operaciones genéricas que se pueden aprender por sí mismas, al margen de cualquier dominio de conocimientos particulares, para luego transferirlos o aplicarse en diferentes contextos. Ser competente en una habilidad o estrategia de pensamiento significa ser capaz de utilizar esa operación útil y eficientemente en una variedad de contextos apropiados. Ser competente en el propio pensamiento crítico implica, entre otras cosas, la adquisición de ciertos tipos de conocimiento, es decir, apropiarse del conocimiento, de ciertos conceptos fundamentales que permitan hacer distinciones, como fundamental en el pensamiento crítico. Mientras que una dificultad del pensamiento crítico es entender la diferencia entre una condición necesaria y suficiente, no como un conocimiento de fondo, sino como la comprensión de los diversos principios que rigen el buen pensamiento en áreas particulares, y muchos de ellos son de dominio específico.

De esta manera, el pensamiento crítico implica algo más que la capacidad de participar en el buen pensamiento. El pensamiento crítico implica el uso de las habilidades que posee el docente. Así, el pensamiento crítico como habilidad supone la existencia de ciertos procesos discretos, procedimientos u operaciones lo que supone que la adquisición de una habilidad implica convertirse en experto en estos procesos. En algunos casos, se cree que estos procesos implican ciertos procesos mentales u operaciones, y en otros, estos procesos se conciben en términos de procedimientos o pasos (Bailin et al., 1999b). 
El uso de la habilidad en este contexto centra la atención en los estudiantes para que sean capaces de un rendimiento inteligente. Alguien que está pensando críticamente puede hacer más que citar una definición. Habilidades que se pueden aprender por sí mismos, al margen de cualquier dominio de conocimiento particular, y luego transferirlo o aplicarlo en diferentes contextos: a) recopilación de información, b) búsqueda de la idea principal, c) determinación de significados (Worsham y Stockton, 1986). Nickerson et al. (1985) indican que es concebible que las personas que poseen los mismos conocimientos puedan diferir de manera significativa en la habilidad con la que aplican lo que saben.

\section{PENSAMIENTO CRÍTICO COMO PROCESO MENTAL}

Un error común en el discurso sobre el pensamiento crítico refiere que ser bueno en el pensamiento crítico es, básicamente, una cuestión de ser competentes en ciertos procesos mentales. Estos procesos generalmente se piensan para incluir cosas tales como la clasificación, la inferencia, la observación, evaluación, síntesis y formulación de hipótesis. Otro error común del pensamiento crítico básicamente se refiere a que se considera como una cuestión de seguir un procedimiento general, en el que se describe generalmente el proceso en términos de un conjunto de pasos, etapas o fases.

Sin embargo, Kirby y Kuykandall (1991) sostienen que pensar es un proceso holístico en que diferentes operaciones mentales trabajan en conjunto. Sin embargo, no existe un consenso acerca de los procedimientos que constituyen el pensamiento, pero se puede considerar en la creación del pensamiento crítico: a) la investigación (método científico), b) la resolución de problemas, y c) la toma de decisiones (Wright, 1993).

Para Marzano et al. (1988), la formación del pensamiento crítico puede darse por conceptos ${ }^{2}$, principios $^{3}$, comprensión $^{4}$, resolución de problemas ${ }^{5}$, toma de decisiones ${ }^{6}$, investigación ${ }^{7}$, composición ${ }^{8}$, y discurso oral ${ }^{9}$. Esto nos da la perspectiva de una evaluación diferente a la que se viene realizando actualmente.

\section{HEURÍSTICA: ESTRATEGIAS, PROCEDIMIENTOS Y DEMÁS}

Para Bailin et al. (1999b) las heurísticas más poderosas tienden a ser aquellos diseñados para que las personas puedan llevar a cabo cada tipo de tareas, en un lugar específico. Son procedimientos diseñados para aplicarse en todos los casos de un pensamiento crítico, como el procedimiento de resolución de problemas. Sin embargo, la investigación psicológica en la resolución de problemas y el funcionamiento cognitivo sugiere una serie de heurísticas simples pero útiles bastante generales. Así, estos autores, creen que la heurística cumple una función útil para aprender a pensar de manera crítica, no lo consideran como la característica central de un buen pensamiento, puesto que tergiversa el principal obstáculo para el buen pensamiento, y groseramente subestima la importancia de los factores contextuales en la decisión de cómo proceder en un caso particular del pensamiento crítico.

En la vista de los procedimientos generales, el desempeño de ciertas tareas se ve que es un medio muy fiable para lograr los resultados deseados de pensar. El reto educativo es, por 
tanto, dotar al alumno de los repertorios de los procedimientos que pueden emplear en toda la gama de situaciones de pensamiento. La realización de tareas tales como el pensamiento de razones a favor y en contra de una posición, o de alternativas de intercambio de ideas, puede garantizar que una persona está pensando críticamente. Las razones en pro y en contra de lo que al individuo se le ocurre pueden abordar sólo los aspectos más triviales de la cuestión; así, también la lluvia de ideas de alternativas puede pasar por alto las más sensatas alternativas.

Aprender a realizar dichas actividades tiene poco mérito educativo, a menos que estas cosas se hagan de una manera tal, que las normas pertinentes de pleno derecho de la adecuación se den. Después de todo, los estudiantes han realizado este tipo de tareas en gran parte de sus vidas. Entonces, el objetivo educativo debe ser el de enseñarles a hacer este tipo de tareas mediante el aumento de su capacidad y ganas de hacer juicios en función de criterios y normas que distinguen las evaluaciones reflexivas, esquemas de clasificación, etc. Es por esta razón que para Bailin et al. (1999a) el pensamiento crítico no debe estar caracterizado en términos de procedimientos que se llevarán a cabo, sino en términos de las normas de una actuación que se debe cumplir para que sea un éxito. En gran medida, lo que debemos hacer para resolver un problema está determinado por las normas que se deben cumplir para la solución y tener éxito.

El pensamiento crítico no se promueve simplemente a través de la repetición de 'habilidades' del pensamiento, entiéndase como la repetición de la teoría en clase; sino a través del desarrollo de los conocimientos pertinentes, los compromisos y las estrategias, y por llegar a entender cuáles son los criterios y las normas relevantes en el entendimiento académico.

\section{HÁBITOS DE LA MENTE}

Como Siegel (1988) menciona que «la tendencia del pensador crítico a las normas y principios de un buen pensamiento no puede simplemente ser el resultado de la habituación». Más bien hay que fomentar la verdadera creencia y la acción responsable, esto es posible si su compromiso es el respeto de los principios y de la verdad, comprometidos con las creencias, valores, acciones y respeto de los productos de alta calidad y prestaciones, acerca del rendimiento activo del buen diseño, y una actitud inquisitiva.

\section{PENSADOR CRÍTICO}

Para Bailin et al. (1999b), el pensamiento crítico es pensar en normas pertinentes, buenas ideas que proporcionan el criterio para determinar que atributos son importantes para los pensadores críticos. Por estas razones, estos autores evitan la conceptualización del pensador crítico en términos que postulan procesos y capacidades mentales o psicológicas. La lista de pensamientos críticos son necesariamente una lista de cosas que el pensador critico debe ser capaz de lograr, es decir, el pensamiento en términos de los resultados generados por el mismo. Por lo tanto, el pensador debe ser capaz de hacer con éxito estas tareas.

Para ser un pensador crítico competente, debe dominar sus propias conceptualizaciones, «eliminar» tal o cual concepto y probar ideas o nombres alternativos (Elder y Paul, 1999, 2001, 2002). Si nos encontramos atrapados en un conjunto de conceptos (ideas, palabras, tradiciones, 
tabúes) pensamos de una manera rígida, lo que nos convierte en incapaces de actuar como personas verdaderamente libres.

En el ámbito educativo, muchos educadores interpretan tales descripciones de habilidad y destreza como descripciones de los procesos psicológicos, estados o capacidades, en lugar de simplemente como descripciones de lo que las personas pueden hacer. Para ello es fundamental la abstracción, la cual recuerda al pensador importantes ideas sobre el aprendizaje. Por esta razón, el educador debe: a) Mirar interrelaciones existentes en la materia dictada, b) Consolidar sus metas de aprendizaje, c) Preguntarse sobre lo que se pretende responder en clase, d) Clarificar la información necesaria en cada clase o en cada pregunta existente, e) Descifrar qué información es trascendente (legitimación de inferencias), f) Buscar y evaluar las implicaciones de nuestro pensamiento, g) Descifrar las ideas clave que ayuden a responder la pregunta o solucionar el problema, h) Asegurar que se adopta el más razonable punto de vista respecto al problema, i) Verificar los supuestos ¿se debe dar por sentado esto o lo otro? (Elder y Paul, 1999, 2001, 2002).

\section{RECURSOS INTELECTUALES NECESARIOS PARA EL PENSAMIENTO CRÍTICO}

Acorde a Bailin et al. (1999a), la mejor manera de caracterizar al pensador crítico es en términos de recursos intelectuales. Estos recursos intelectuales son de tres clases:

- El conocimiento previo. El pensamiento crítico siempre tiene lugar en el contexto ya existente, con creencias, valores y formas de actuar, por lo tanto, la profundidad del conocimiento y entendimiento de la experiencia de las personas tienden a ser de un estudio particularmente significativo. Del mismo modo, el pensamiento crítico rechaza el juicio a la moral, por lo tanto, requiere una comprensión clara de la naturaleza de la acción o política de ser juzgado el contexto en el que se llevan a cabo, y la gama de consideraciones morales pertinentes en el juicio.

- El conocimiento operativo de las normas de buen pensamiento. Las normas pertinentes de evaluación crítica en el desempeño de las tareas de pensamiento están en el corazón del pensamiento crítico. Por lo tanto, el conocimiento a nivel operativo de las normas que rigen la deliberación crítica y el juicio, hace necesario para cualquier persona pensar críticamente. Para tal efecto, es útil tener en cuenta las normas que comprende dos tipos: 1) Las normas que son relevantes para los intelectuales, así tenemos: argumentos, teorías, juicios legales, trabajos de arte, y 2) Las prácticas de deliberación o consulta. Normas y principios implícitos en las buenas practicas criticas funcionan en un hablar y escribir correctamente, sin ser capaz de establecer los estándares de buen uso de la lengua. También se puede aprender a pensar de manera crítica, sin poner estándares de pensa-miento crítico.

- El conocimiento de los conceptos críticos claves. El pensamiento crítico implica evaluar los problemas intelectuales; es decir, argumentos, declaraciones, criterios y normas pertinentes a la evaluación de los productos intelectuales confrontado con una declaración que requiere una evaluación, que tendrá que reconocer si se trata de una declaración de valor, una evaluación empírica, o una declaración conceptual para que puedan hacer una evaluación 
responsable de la misma. Los conceptos permiten al pensador crítico evaluar fácilmente, este debe tener concepto crítico como premisa o conclusión sin perder estos términos.

\section{TAREA DE ENSEÑAR EL PENSAMIENTO CRÍTICO}

El bien primario de una buena concepción del pensamiento crítico y el pensador crítico es el de proveer concretamente conocimientos, algo complementario en la enseñanza del pensamiento crítico. Bailin et al. (1999a) indican que «la enseñanza del pensamiento crítico es en gran medida una cuestión de enseñar a los estudiantes los conceptos para el uso apropiado de normas establecidas y procedimientos culturales disciplinarios». Por lo tanto, la enseñanza de habilidades y disipaciones aisladas, son más bien como la promoción de la iniciación de los alumnos en prácticas criticas complejas que incorporan valor, compromisos y requieren el uso sensible de una variedad de recursos intelectuales en el ejercicio del buen juicio. Aunque el proyecto educativo a largo plazo es desarrollado en el pensamiento crítico en una gran variedad de áreas, la constitución de este fin es necesariamente un proceso que puede comenzar en los primeros años en la escuela.

El éxito en ayudar a los profesores a ver lo que está involucrado en la enseñanza del pensamiento crítico se puede describir con la participación de tres componentes: a) Involucrar a los estudiantes para hacer frente a las tareas que requieren razonamiento, juicio o evaluación, b) Ayudar a desarrollar recursos intelectuales para hacer frente a estas tareas, y c) Proporcionar un entorno en el que pensamiento crítico es evaluado y se anima, para ello, el apoyo a los estudiantes en sus intentos para pensar críticamente y participar en la discusión. En este sentido, un buen pensamiento debe interpretarse en ausencia de evidencia empírica.

Bailin et al. (1999b) señalan que para aquellos interesados en enseñar a los estudiantes a ser mejores en el pensamiento crítico, no se puede enseñar a los estudiantes el proceso de notar falacias, ya que no hay motivo para creer que hay un proceso de este tipo. Lo más que pueden hacer por ellos es orientar, y esto, al parecer, se lo puede hacer en al menos tres formas: a) Enseñamos a las personas ciertos conceptos, por ejemplo, el concepto de un argumento válido; esto les permite darse cuenta de las falacias que de otro modo habrían pasado por alto, pero no garantiza de que se darán cuenta de ello, b) Motivamos al estudiante para que cuide de que los argumentos sean válidos y estar al pendiente de los argumentos no válidos, y c) Enseñamos procedimientos que permiten a la persona orientarse a sí misma cuando se buscan ciertos tipos de recepción. En este ámbito, a nivel curricular se requiere de los estudiantes: 1) Analizar, sintetizar y evaluar, y 2) Creación de nuevos productos como ensayos (orales y escritos) y creaciones artísticas.

\section{CONCLUSIONES}

El pensamiento crítico es una actividad de vida, no un pasatiempo académico abstracto. Es algo que todos hacemos a pesar de su frecuencia, y la credibilidad que le otorgamos varía de persona a persona. Por esta razón, el pensamiento crítico ha sido interpretado de diversas maneras. Se ha equiparado con el desarrollo de habilidades de razonamiento lógico, con la aplicación de juicio reflexivo, y con la creación, uso y pruebas de significación. 
El pensamiento crítico es generalmente concebido como una capacidad intelectual adecuada para el desarrollo de las personas involucradas en los estudios empíricos más allá del desarrollo de las capacidades de pensamiento crítico. Esto ha permitido generar interpretaciones. Una interpretación alternativa del concepto de pensamiento crítico es el del aprendizaje emancipador, es decir, aquel que salga de la lógica tradicional del aprendizaje para que se base en la discusión y el diálogo. Otra interpretación es el pensamiento dialéctico visto como una forma particular de pensamiento crítico que se centra en la comprensión y resolución de contradicciones, es decir, la aplicación heurística que sugieren examinar las consecuencias positivas y negativas de todas las alternativas conocidas, incluyendo las inaceptables.

Para la aplicación del pensamiento crítico en clase, se debe ser un pensador crítico; esto implica más que las actividades cognitivas como el razonamiento lógico o examinar argumentos a favor de afirmaciones no respaldadas por pruebas empíricas. Pensar críticamente implica nuestro reconocimiento de los supuestos que subyacen a nuestras creencias y comportamientos. Por tanto, se requiere recursos intelectuales, mismos que son de tres clases: a) El conocimiento previo, b) El conocimiento operativo de las normas de buen pensamiento, y c) El conocimiento de los conceptos críticos claves.

\section{NOTAS}

1 En la mayoría de los casos, las evaluaciones se circunscriben al ámbito de las calificaciones por respuestas obtenidas, y en ese sentido, la generación del pensamiento crítico se detiene.

2 Construcciones o imágenes mentales por medio de las cuales comprendemos las experiencias que emergen de la interacción con nuestro entorno. Aquí es fundamental la discusión de las diversas escuelas económicas.

3 Reglas y normas de la construcción en la teoría económica.

4 Percibir las cosas como realmente son. Se lo puede lograr mediante lecturas, análisis de caso, determinando el problema y las soluciones posibles.

5 Conclusión de un proceso, mediante propuestas alternativas a lo que se viene realizando en el proceso económico.

6 Proceso de elección de opciones mediante la discusión de la política pública o del mercado, etc.

7 Estrategia para descubrir algo mediante la búsqueda de bibliografía para trabajos o ensayos.

8 Formación de un todo mediante la realización de ensayos que construyan un discurso plural de las distintas visiones.

9 Sucesión de palabras de forma oral, mediante la presentación grupal o individual de los trabajos realizados o temáticos considerados en los syllabus.

\section{BIBLIOGRAFÍA}

Bailin, S. Case, R. Coombs, J. y Daniels, L (1999a). Common misconceptions of critical thinking. Journal of Curriculum Studies, 31(3), 269-283.

Bailin, S. Case, R. Coombs, J. y Daniels, L (1999b). Conceptualizing critical thinking. Journal of Curriculum Studies, 31(3), 285-302.

Brookfield, S. D. (1987). What It Means to think Critically. In S. D. Brookfield (Ed.), Developing Critical Thinkers: Challenging Adults To Explore Alternative Ways of Thinking and Acting (p. 3-14). San Francisco, CA: Jossey-Bass. 
Elder, L. y Paul. R. (1999). Critical thinking: Teaching students to seek the logic of things. Journal of developmental education, 23(1), 34-35.

Elder, L. y Paul. R. (1999). Critical thinking: Teaching students to seek the logic of things, Part II. Journal of developmental education, 23(2), 34-35.

Elder, L. y Paul. R. (2001). Critical thinking: Thinking with concepts. Journal of developmental education, 24(3), 42-43.

Elder, L. y Paul. R. (2002). Critical thinking: Distinguishing between inferences y assumptions. Journal of developmental education, 25(3), 34-35.

Kirby, D. y Kuykendall, C., (1991). Mind Matters: Teaching for Thinking. Portsmouth, NH: Boynton/Cook. Marzano, R. J., Brandt, R. S., Hughes, C. S., Jones, B. F., Presseisen, B. Z., Rankin, C. S. y Suhor, C. (1988) Dimensions of Thinking: A Framework for Curriculum and Instruction. Alexandria, VA: Association for Supervision and Curriculum Development.

Nickerson, R. S., Perkins, D. N. y Smith, E. E., (1985). The Teaching of Thinking. Hillsdale, NJ: Erlbaum.

Siegel, H. (1988). Educating Reason: Rationality, Critical Thinking and Education. London, England: Routledge.

Worsham, A. M. y Stockton, A. J. (1986). A Model for Teaching Thinking Skills: The Inclusion Process. Fastback 236. Bloomington, IN: Phi DeltaKappa.

Wright, I. (1993). Inquiry, problem-solving, and decision making in elementary social studies methods textbooks. Journal of Social Studies Research, 16-17(1), 26-32. 
